\section{BLM Analyzer: a software tool for experiments on planar lipid bilayers}

\author{
Pavel Novák, Marta Gaburjáková, and Ivan Zahradník \\ Slovak Academy of Sciences, Bratislava, Slovakia \\ BioTechniques 42:335-341 (March 2007) \\ doi 10.2144/000112384
}

\begin{abstract}
Planar lipid bilayers represent a versatile platform for studying the functions of various membrane proteins as well as the development of biosensors. Despite the continuing technological progress in the fabrication of low-noise bilayer setups with mechanically and electrically stable planar bilayers, there is still a lack of software utilities for assistance during bilayer formation. We present here a multipurpose software tool, the bilayer lipid membrane (BLM) Analyzer, which performs high-resolution measurements of bilayer capacitance and resistance using saw-tooth voltage stimulation. Based on the measured values of capacitance and resistance, the BLM Analyzer detects formation, stabilization, and breakage of lipid bilayer, automatically selects appropriate stimulus protocol, compensates for voltage offsets, and issues sound and voice alerts informing about the state of the measurement cycle. The principle of the BLM Analyzer is based on the integration of current responses within four equivalent time segments. It provides capacitance estimates with standard deviation of several femtofarads at temporal resolution of several tens of milliseconds. The functions of the BLM Analyzer were tested experimentally by monitoring formation and thinning of planar lipid bilayer.
\end{abstract}

\section{INTRODUCTION}

Planar bilayer membranes formed over a small aperture in a partition separating two compartments represent a versatile platform for studies of membrane ion channels (1-3), membrane fusion (4), as well as the development of biosensors (5). The method of the planar bilayers allows controlling precisely the composition of solutions on both sides of the bilayer, as well as the composition of the bilayer itself. Ion channels from intracellular organelles (such as sarcoplasmic reticulum or mitochondria) not directly accessible to the patch-clamp technique can be incorporated into the bilayer and investigated under precisely defined experimental conditions. The limiting step in the effectiveness of the method is its high demand of labor skills and attention. Several promising solutions to the problem of aperture fabrication for low-noise recordings $(1,6,7)$ and reduction of the time required to form lipid-bilayer have been introduced (8). Despite the continuing progress in bilayer technology, software utilities
A

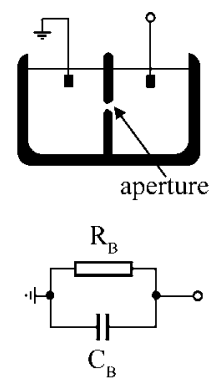
transition glitches. resulting square wave current response is proportional to the value of bilayer capacitance. Users of PULSE and XCHART (www.heka.com) are able run the protocol and macro files developed originally for the two-electrode voltage clamp measurement of membrane capacitance using the paired-ramps stimulus protocol (10); however there is no such opportunity for users of other popular systems, such as the pClamp and Digidata 132x family of data acquisition systems (www.moleculardevices. com). To fill this gap in the software support of bilayer experiments, we have developed a multipurpose software tool that features real-time, high-resolution analysis of capacitance and resistance of lipid bilayer as well as other useful automated functions, such as sound alerts informing about bilayer formation, thinning, and breakage, and automatic zeroing of voltage offsets prior to membrane formation. This software, called bilayer lipid membrane (BLM) Analyzer, integrates current responses within specified time segments (11) to provide high-resolution capacitance estimation in a way similar to the previous report using the unipolar voltage stimulus consisting of two consecutive ramps of inverse slope (10). We have developed this idea further and extended the algorithm to use with bipolar sawtooth voltage stimulus, allowing highresolution measurement of capacitance, resistance, and leak current at the same time with the additional advantage of
B

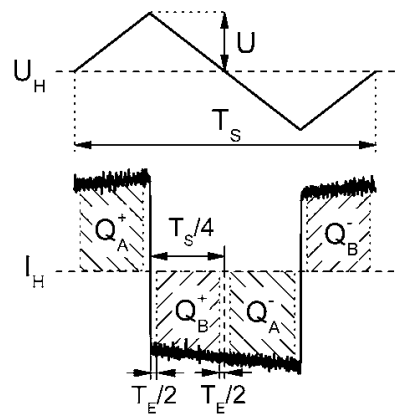

Figure 1. Principle of the planar lipid bilayer technique and the BLM Analyzer. (A) The measurement chamber for bilayer experiments (top) can be modeled by a parallel combination of the resistance $R_{B}$ and the capacitance $C_{B}$ (bottom) in series with the access resistance $R_{A}$. (B) The voltage stimulus (top) used for monitoring of the bilayer formation consists of the symmetrical bipolar saw-tooth wave, of the amplitude $U$, and the stimulation period $T_{S}$ superimposed on the holding potential $U_{H}$. Integration of the current response (bottom) within each quarter of the stimulus period yields four charges: $Q_{A^{+}}, Q_{B}{ }^{+}, Q_{A}{ }^{-}$, and $Q_{B}$. The small time interval $T_{E}$ is excluded from the integration in each quarter-period to suppress the effect of filtering or 
improved rejection of power line interference.

\section{MATERIALS AND METHODS}

\section{Principle of the Measurement}

The measurement chamber for bilayer experiments with small a aperture in the separating wall can be represented as parallel combination of capacitance $C_{B}$ and resistance $R_{B}$ (Figure 1A). Prior to the formation of the bilayer, $C_{B}$ corresponds to the small parasitic capacitance of the separating wall, and $R_{B}$ corresponds mostly to the resistance of the aperture filled with electrolyte solution (3). After formation of the bilayer, $C_{B}$ represents the capacitance of the bilayer, and $R_{B}$ represents the resistance of the bilayer. The access resistance, which includes the resistance of electrodes, salt bridges, bath solution, and solution within the aperture, is negligible compared with bilayer resistance (3) and can be ignored when calculating $C_{B}$ and $R_{B}$. The process of bilayer formation can be monitored by measuring the current $i_{B}(t)$ flowing through the chamber during the bipolar symmetrical sawtooth voltage stimulation $v(t)$ (Figure 1B). Since the current through the capacitance is $i_{C}=C_{B} \times d v / d t$, while the current through resistance is simply $i_{R}$ $=v / R_{B}$, the resulting $i_{B}(t)$ consists of the square wave current component with amplitude $4 U C_{B} / T_{S}$ flowing through the capacitance and the saw-tooth current component with amplitude $U / R_{B}$ flowing through the resistance, where $U$ stands for the amplitude of the voltage stimulus and $T_{S}$ stands for the stimulus period (Figure 1B). Integration of the current response $i_{B}(t)$ within specific time segments provides charges $Q_{A^{+}}, Q_{A}{ }^{-}, Q_{B}{ }^{+}$, and $Q_{B}{ }^{-}$(Figure $1 B)$. The value of each of these charges is simply the area of trapezoid (Figure 1B), which can be calculated as average current within the given time segment multiplied by the width of the time segment:

$$
Q_{A}^{+}=-Q_{A}^{-}=\left(\frac{4 U C}{T_{S}}+\frac{U}{2 R_{B}}+I_{H}\right)\left(\frac{T_{S}}{4}-T_{E}\right)
$$

[Eq. 1]
$Q_{B}^{+}=-Q_{B}^{-}=\left(-\frac{4 U C}{T_{S}}+\frac{U}{2 R_{B}}+I_{H}\right)\left(\frac{T_{S}}{4}-T_{E}\right)$

[Eq. 2]

where $T_{E}$ represents the time interval excluded from integration and $I_{H}$ represents the holding current (Figure 1B). Similarly to the previous report (10), the capacitance and resistance can be calculated as:

$$
\begin{gathered}
C_{B}=\frac{Q_{A}-Q_{B}}{2 U} \frac{T_{S}}{T_{S}-4 T_{E}} \\
R_{B}=\frac{U\left(\frac{T_{S}}{4}-T_{E}\right)}{Q_{A}+Q_{B}}
\end{gathered}
$$

where the charges $Q_{A}$ and $Q_{B}$ are defined as:

$$
\begin{aligned}
& Q_{A}=\left(Q_{A^{+}-} Q_{A^{-}}\right) / 2 \\
& Q_{B}=\left(Q_{B^{+}-} Q_{B}{ }^{-}\right) / 2
\end{aligned}
$$

In addition to values of $R_{B}$ and $C_{B}$, it is possible to calculate the holding current $I_{H}$ at holding potential $U_{H}$ simply as the mean current value within stimulus period $T_{S}$ :

$$
I_{H}=\left(Q_{A^{+}}+Q_{B^{+}}+Q_{A^{-}}+Q_{B^{-}}\right) / T_{S}
$$

Thanks to the bipolar character of the voltage stimulus, harmonic signals (sinusoidal or cosinusoidal) with period $T_{I}=T_{S} /(2 n)($ where $n=1,2,3$, etc.) are automatically suppressed by the averaging of charges in Equations $5-7$. If these charges are calculated from the current response obtained from the average of $m$ consecutive current responses, then every harmonic signal with a period of $T_{I}=m T_{S} /(2 n)$ is suppressed. This is a very useful feature considering the permanent problem of power line interference in highly sensitive bilayer setups.

\section{Detection of Steps in Bilayer Formation, Thinning, and Breakdown}

Prior to formation of the bilayer, the measured electrical resistance $R_{B}$ is relatively low (between $1-100 \mathrm{k} \Omega$ ) depending on the geometry of aperture and the specific electrolyte resistance (3), as well as the capacitance $C_{B}$, which is of only few picofarads $(\mathrm{pF})$, depending on the material and geometry of the separating wall. After painting the lipid solution over the aperture, both the resistance $R_{B}$ and capacitance $C_{B}$ are increased by at least one order of magnitude as the bilayer forms. Automatic detection of the bilayer formation is based on the detection of a nonrandom increase in $R_{B}$ above the user-specified value. The initial phase of bilayer formation is usually followed by a gradual thinning of the bilayer, which is reflected as the slowly increasing bilayer capacitance (8). To facilitate observation of this process, a time derivative of membrane capacitance $d C_{B} / d t$ is evaluated and displayed along with values of $C_{B}$ and $R_{B}$. Termination of the bilayer thinning is detected simply as a decrease of $d C_{B} / d t$ below the user-specified value during the user-specified time interval. A sudden nonrandom drop of bilayer resistance below the user-defined value any time after the start of bilayer formation is recognized as the bilayer breakdown.

\section{Development of BLM Analyzer}

Algorithms described in the sections entitled Principle of the Measurement and Detection of Steps in Bilayer Formation, Thinning, and Breakdown were implemented in the 32-bit Microsoft ${ }^{\circledR}$ Windows ${ }^{\circledR}$ application BLM Analyzer using Visual $\mathrm{C}++^{\circledR}$. ActiveX ${ }^{\circledR}$ chart control NTGraph (www.codeproject.com/miscctrl/ ntgraph_activex.asp) was used for the display of the current response waveform and the time courses of the measured capacitance and resistance values. The measured parameter values are saved in Axon Binary File (ABF) file format using the pClamp file support pack (www.moleculardevices. 


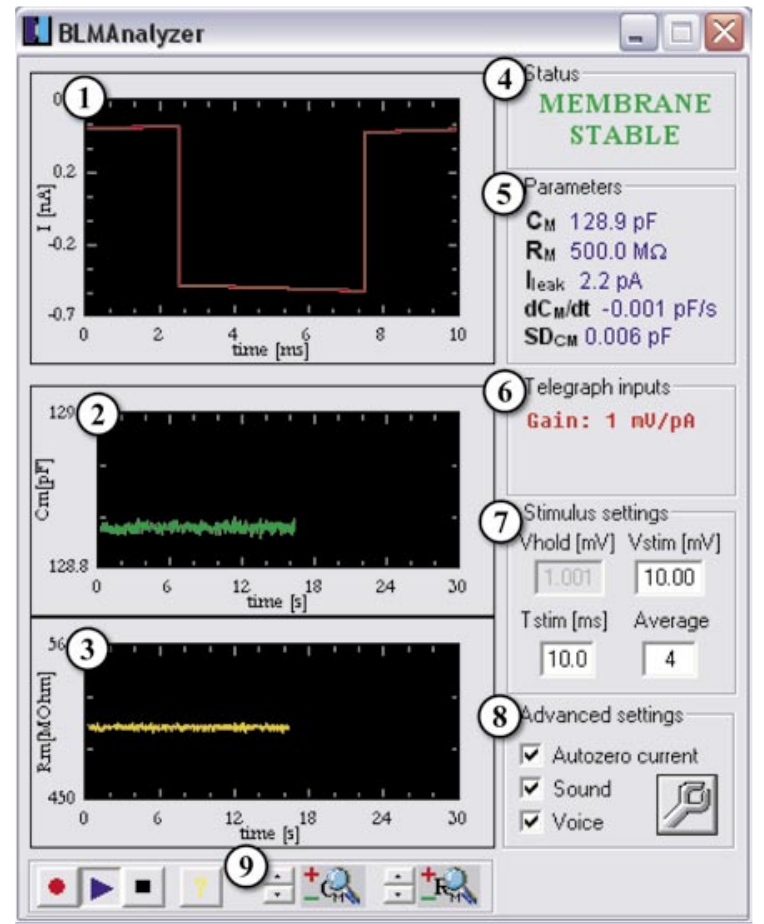

com/pages/software/developer_info. html).

\section{Electrophysiological Recordings}

BLM Analyzer was tested using both the bilayer model cell MCB-1U (Axon Instruments/Molecular Devices, Sunnyvale, CA, USA), consisting of a $10-\mathrm{k} \Omega$ resistor in series with a 100 $\mathrm{pF}$ capacitor, and the real planar lipid bilayers. Model currents were measured with Axopatch 200B amplifier (Axon Instruments/Molecular Devices) in the voltage-clamp mode and digitized using a Digidata 1320A (Axon Instruments/Molecular Devices). The saw-tooth voltage amplitude was set to $10 \mathrm{mV}$ (i.e., $20 \mathrm{mV}$ peak-to-peak), and the stimulation period $T_{S}$ was from 40-5 ms. The number of averaged measurement cycles (average ratio $m$ ) was adjusted to keep the product $m . T_{S}$ constant and equal to $40 \mathrm{~ms}$. The Axopatch again was set to $1 \mathrm{mV} / \mathrm{pA}$, and the current response was filtered using the built-in $10-\mathrm{kHz}$ low-pass filter. When testing the accuracy of the BLM Analyzer, a 500-M $\Omega$ resistor was added in parallel to the capacitor in the MCB-1U model, and the original capacitor was replaced by 22,47 , and $150 \mathrm{pF}$ capacitors. All
Figure 2. The BLM Analyzer dialog screen. Information about the currently running measurement is represented both in the graphical form (1, the current response waveform; 2 , the time course of the measured capacitance $C_{B}$; and 3 , the resistance $R_{B}$ ) and the textual form (4, the state of measurement; 5 , current values of parameters; and 6 , telegraph inputs). The user may quickly modify properties of the voltage stimulus, 7 , to switch the auto-zeroing and sound alerts, 8 , on or off to start or stop the measurement, or to zoom in or out the trace of measured parameters, 9 .

capacitors and resistors were of the $5 \%$ tolerance grade. Capacitance and resistance of all used components were measured independently by a BM 595 digital resistance-inductance-capacitance (RLC) bridge meter (TESLA, Liptovsky, Hradek, Slovakia).

Planar lipid bilayers were formed by painting the 3:1 phosphatidyl ethanolamine/phosphatidyl serine (Avanti Polar Lipids, Alabaster, AL, USA) mixture onto the aperture in partition wall. The bilayer cup was made of polystyrene. Bilayer current recordings were done with the BC-525 bilayer clamp amplifier (Warner Instruments, Hamden, CT, USA) and digitized with the Digidata 1322A. The amplifier gain was set to $100 \mathrm{mV} / \mathrm{pA}$, and the built-in $10 \mathrm{kHz}$ low-pass filter was selected. The sawtooth amplitude was limited to $5 \mathrm{mV}$ to prevent amplifier and digitizer overload. The stimulus period was fixed to $10 \mathrm{~ms}$, and the average ratio to $m=4$.

\section{RESULTS AND DISCUSSION}

\section{Software Description}

The BLM Analyzer features a userfriendly graphical interface (Figure 2) based on a single dialog window encompassing all controls essential for the real-time measurement. The BLM Analyzer continuously evaluates the current response and estimates the capacitance, the resistance and the holding current, and automatically detects three distinct measurement states: (i) no membrane, (ii) membrane formation, and (iii) membrane stable. Criteria for transitions between the three states can be modified in a separate dialog box. The user is notified both visually (by colored text controls) and acoustically regarding changes in the measurement status. Voice alerts inform the user of current values of measured parameters. Besides serving as a basis for user notifications about the state of the bilayer formation, the identification of the current measurement status is also used for the automated setting of the voltage stimulus amplitude and holding potential (according to the user-defined values) and automated deactivation of the auto-zeroing upon detection of the membrane formation. Automated settings and sound notifications reduce the need for user intervention and frequent visual inspection of the monitor screen, therefore saving time for other tasks accompanying experiments on lipid bilayers.

Currently, there is no other software tool to which the BLM Analyzer could be directly compared, especially when it comes to the measurements using the Digidata132x family of data acquisition systems. Previously published implementation of capacitance measurement based on paired voltage ramps (10) relies on PULSE and X-CHART programs without any support for Digidata 132x. Other commercial tools supporting Digidata $132 \mathrm{x}$, such as pClamp, jClamp (www.scisoftco.com/jclamp. html), or freely available Strathclyde Electrophysiology software (spider. science.strath.ac.uk/PhysPharm/ showPage.php? pageName=software), offer various versions of seal tests or membrane tests based on the squarewave voltage stimulation. These are not suitable for capacitance measurements on planar bilayers due to the very short charging time constant of bilayer capacitance (usually below the sampling period) and the large peak 


\section{Short Technical Reports}
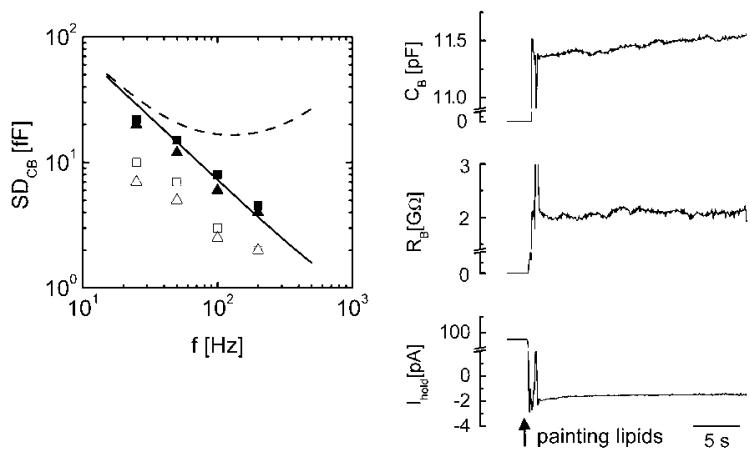

Figure 3. The resolution of capacitance measurement. (A) The standard deviation (SD) of capacitance $C_{B}$ as a function of the stimulation frequency $f_{S}$ measured by BLM Analyzer on the bilayer model cell MCB-1U with $C_{B}=100 \mathrm{pF}$ (squares) or $10 \mathrm{pF}$ (triangles) and Axopatch 200B headstage in the resistivefeedback mode (solid symbols) or the capacitor-feedback mode with active cooling (hollow symbols). The solid line represents the theoretical prediction for bilayer model MCB-1U with $C_{B}=100 \mathrm{pF}$ calculated for high-resolution sine-wave method (Equation 14 in Reference 13). The dashed line represents the theoretical prediction for the same bilayer model with original access resistance of $10 \mathrm{k} \Omega$ replaced by $5 \mathrm{M} \Omega$ to simulate conditions typical for whole-cell patch clamp experiment. (B) Typical records of the bilayer capacitance $C_{B}$, resistance $R_{B}$ and holding current $I_{\text {hold }}$ during formation of the planar lipid bilayer. The standard deviation of the capacitance estimation was $10 \mathrm{fF}$.

current, both resulting from low-access resistance of conventional bilayers (3). Since the BLM Analyzer is a fully autonomous program not tied to any software environment, it is possible to run it side-by-side with any of the above mentioned electrophysiology software packages, provided that only one of them acquires data from Digidata 132x. Measured values of capacitances, resistances, and holding currents can be recorded and stored in real-time in $\mathrm{ABF}$ format together with original current responses (Figure 1B) for later analysis in pClamp or other tools supporting $\mathrm{ABF}$ format, such as Origin (OriginLab, Northampton, MA, USA) or SigmaPlot (Systat Software, Point Richmond, CA, USA).

When compared with Warner bilayer clamp amplifiers (BC-525 and $\mathrm{BC}-535$; www.warneronline. com) or the Dagan 3900 amplifier with bilayer expander module (www. dagan.com/3900a.htm) -all models based on built-in hardware capacitance meters-the BLM Analyzer clearly outperforms both of them. These hardware tools serve perfectly for basic checking of bilayer presence or absence and for quick information about the instant value of bilayer capacitance. However, they do not provide any information about the bilayer resistance, do not offer recording of the time course of measured parameters, nor do they provide variable temporal resolution of the capacitance measurement.

The BLM Analyzer is freely available (see the supplementary material available at www.BioTechniques.com for installation file). Fully commented $\mathrm{C}++$ source code for calculation of the capacitance, resistance, and leak current and for the automatic detection of bilayer formation, thinning, and breakage is included in the installation of BLM Analyzer so that users of digitizers other than the Digidata $132 x$ family of data acquisition systems can adapt the code easily.

\section{Resolution of BLM Analyzer}

The BLM Analyzer is able to run at high stimulation rates (up to several kilohertz with a $500-\mathrm{MHz}$ Intel ${ }^{\circledR}$ Pentium $^{\circledR}$ processor) and allows averaging of multiple stimulation cycles-a strategy commonly used for high-resolution capacitance measurement $(11,12)$. The smallest resolvable capacitance change defining the resolution of the measurement method is usually determined by the steady-state standard deviation of capacitance estimation $\left(\mathrm{SD}_{C B}\right)$. As illustrated in Figure $3 \mathrm{~A}, \mathrm{SD}_{C B}$ below $10 \mathrm{fF}$ could be obtained already at a moderate stimulation frequency $(100 \mathrm{~Hz})$ and a low averaging degree (only four stimulation cycles). With the actively cooled headstage of the Axopatch-200B in capacitor-feedback mode, the same resolution could be obtained even at four times lower stimulation frequency without any averaging (Figure 3A). The standard deviation of capacitance measured with the BLM Analyzer was virtually independent of the value of capacitor used in the bilayer model (Figure 3A). Similar independence was observed in previously published works using the paired voltage ramps (10). Capacitance resolution seemed to be determined mainly by the background noise originating in the amplifier headstage, the leak resistance of the capacitor, and other mechanical and electrical parts of the measurement setup. Given that the root mean square (RMS) value of current noise $I_{R M S}$ measured in frequency bandwidth $B_{R M S}$ reported by the digital RMS meter of the amplifier, one may calculate expected value of $\mathrm{SD}_{C B}$ simply as:

$$
\mathrm{SD}_{C B}=I_{R M S} \cdot T_{S}^{0.5 /(8 U) /\left(m \cdot B_{R M S}\right)^{0.5}}
$$

[Eq. 8]

where $m$ represents the average ratio. Thanks to the virtual independence of $\mathrm{SD}_{C B}$ on the value of $C_{B}$, the attainable capacitance resolution in bilayer experiments can be significantly better than in the whole-cell patch-clamp technique operating in the similar range of membrane capacitances (Figure 3A). The better capacitance resolution of the bilayer technique originates basically in much lower access resistance of the bilayer technique in comparison with typical access resistance of the wholecell patch-clamp technique (3).

Experiments on real planar lipid bilayers proved the high performance and reliability of the BLM Analyzer. The bilayer formation (Figure 3B) and the bilayer breakdown states were correctly and reliably recognized by BLM Analyzer in all performed experiments. Low standard deviation of bilayer capacitance estimates $\left(\mathrm{SD}_{C B}\right.$ $=10 \mathrm{fF}$ at $40 \mathrm{~ms}$ temporal resolution) allowed the monitoring of the gradual increase of bilayer capacitance at the rate of $10 \mathrm{fF} / \mathrm{s}$ during bilayer thinning immediately after bilayer formation (Figure 3B). Considering the fact that these measurements were performed with a stimulation amplitude of only 5 $\mathrm{mV}$, the standard deviation $\mathrm{SD}_{C B}$ of $10 \mathrm{fF}$ 
reported here corresponds to $5 \mathrm{fF}$ under conditions of the model measurement (see Equation 8 for effect of stimulus amplitude). Even with the open front door of the Faraday cage enclosing the bilayer setup (when the power line interference component in the current output had $5 \mathrm{pA}$ amplitude) the standard deviation was still relatively low $(<20$ fF) thanks to the inherent rejection of the power-line interference. The superior noise performance of the BLM Analyzer is obvious just from visual comparison of our records with recently published measurements of capacitance during bilayer thinning (see Figure 3B in Reference 8), where the relative peak-to-peak noise of the capacitance trace is around $10 \%$ of the capacitance value; in our work, it is only $0.4 \%$.

The algorithm of the BLM Analyzer for calculation of charges $Q_{A}$ and $Q_{B}$ (Equations 5 and 6) acts like a band-pass filter (see Reference 11 for details on the transfer function of such an algorithm), inherently rejecting any nonperiodic, multispectral noise, including the noise resulting from mechanic and acoustic vibrations of the setup, although not as efficiently as periodic power line interference. The effect of noise on capacitance measurements is described by Equation 8 and can be decreased by using a higher average ratio $m$ combined with shorter stimulus period $T_{S}$ or higher stimulus amplitude $U$.

\section{Accuracy of the BLM Analyzer}

Any measurement method can be referred to as high-resolution and high-precision only if the output results are highly reproducible (the standard deviation is as low as possible) and the mean value of output results is as close as possible to the real absolute values of the measured parameters. Our model measurements revealed that the mean value of capacitance reported by the BLM Analyzer laid within the $5 \%$ tolerance band of capacitors and differed by $<1.5 \%$ from values reported by the digital RLC bridge meter. Accuracy of the capacitance measurement was not significantly affected the by addition of the $500-\mathrm{M} \Omega$ resistor representing bilayer resistance, nor by the presence or absence of the series $10-\mathrm{k} \Omega$ resistor representing resistance of electrodes and electrolyte. The value of the bilayer resistance reported by the BLM Analyzer differed by no more than $2.3 \%$ from the value measured by the RLC meter in all tested model configurations. We conclude that the BLM Analyzer provides both accurate and highly reproducible estimates of bilayer capacitance and resistance.

\section{Conclusions}

Thanks to automation capabilities combined with the high-resolution capacitance measurement, the BLM Analyzer may serve both beginners mastering the bilayer technique, as well as experienced experimenters interested in studies of membrane thinning, vesicle fusion, and other membranerelated processes reflected in bilayer capacitance or conductance. A possible future development of the BLM Analyzer includes implementation of the automatic detection of vesicle fusions during ion channel reconstitutions, implementation of the continuous recorder for the single channel current recordings, and the support for other data acquisition systems.

\section{ACKNOWLEDGMENTS}

This work was supported by grants from the Slovakia Science and Technology Assistance Agency (APVT) 51-031104 and the Slovakia Scientific Grant Agency (VEGA) 2/4153/04 to I.Z.

\section{COMPETING INTERESTS STATEMENT}

The authors declare no competing interests.

\section{REFERENCES}

1.Pantoja, R., D. Sigg, R. Blunck, F. Bezanilla, and J.R. Heath. 2001. Bilayer reconstitution of voltage-dependent ion channels using a microfabricated silicon chip. Biophys. J. 81:2389-2394.

2.Woodbury, D.J. and C. Miller. 1990. Nystatin-induced liposome fusion. A versatile approach to ion channel reconstitution into planar bilayers. Biophys. J. 58:833-839.

3. Wonderlin, W.F., A. Finkel, and R.J. French. 1990. Optimizing planar lipid bilayer single-channel recordings for high resolution with rapid voltage steps. Biophys. J. 58:289297.

4. Cho, S.J., M. Kelly, K.T. Rognlien, J.A. Cho, J.K. Horber, and B.P. Jena. 2002. SNAREs in opposing bilayers interact in a circular array to form conducting pores. Biophys. J. 83:2522-2527.

5. Bayley, H. and P.S. Cremer. 2001. Stochastic sensors inspired by biology. Nature 413:226230.

6. Mayer, M., J.K. Kriebel, M.T. Tosteson, and G.M. Whitesides. 2003. Microfabricated teflon membranes for low-noise recordings of ion channels in planar lipid bilayers. Biophys. J. 85:2684-2695.

7.Fertig, N., C. Meyer, R.H. Blick, C. Trautmann, and J.C. Behrends. 2001 Microstructured glass chip for ion-channel electrophysiology. Phys. Rev. E. Stat. Nonlin. Soft. Matter Physiol. 64:040901.

8. Ide, T. and T. Ichikawa. 2005. A nove method for artificial lipid-bilayer formation. Biosens. Bioelectron. 21:672-677.

9. Novak, P. and I. Zahradnik. 2005 Computer-aided formation of the whole-cell patch-clamp recording configuration. Gen. Physiol. Biophys. 24:337-348.

10.Schmitt, B.M. and H. Koepsell. 2002. An improved method for real-time monitoring of membrane capacitance in Xenopus laevis oocytes. Biophys. J. 82:1345-1357.

11. Novak, P. and I. Zahradnik. 2006. Qmethod for high-resolution, whole-cell patch-clamp impedance measurements using square wave stimulation. Ann. Biomed. Eng. 34:1201-1212.

12. Gillis, K.D. 1995. Techniques for membrane capacitance measurements, p. 155-198. In B. Sakmann and E. Neher (Eds.), Single-Channel Recording. Plenum Press, New York.

13. Chen, P. and K.D. Gillis. 2000. The noise of membrane capacitance measurements in the whole-cell recording configuration. Biophys. J. 79:2162-2170

Received 4 August 2006; accepted 1 November 2006.

Address correspondence to Pavel Novák, Institute of Molecular Physiology and Genetics, Slovak Academy of Sciences, Vlárska 5, 83334 Bratislava, Slovak Republic.e-mail:pavel.novak@savba.sk

To purchase reprints of this article, contact: Reprints@BioTechniques.com 